\title{
Study on Decision-Making Model of Bid Evaluation for Engineering Projects Based on BP Neural Network and Order Relation Analysis
}

\author{
Yi Zhang ${ }^{1,2, *}$ \\ ${ }^{I}$ School of Management, Hubei University of Technology, Wuhan, Hubei, P.R. China \\ ${ }^{2}$ Collaborative Innovation Centre for Regional Eco-industry Development, Hubei University of Technology, Wuhan, \\ Hubei, P.R. China
}

\begin{abstract}
Using the theory and method of artificial neural network, this paper established a decision-making model of bid evaluation for engineering projects based on back propagation neural network (BPNN). BP neural network was trained and tested by the learning sample that was attained by using order relation analysis method $\left(\mathrm{G}_{1}\right.$-method). At the same time, we used the momentum BP algorithm to solve the model. The results show that the model is feasible and reasonable, possessing high abilities of self-organization, self-adaptation and self-learning and strong function of fault-tolerance, avoiding weaknesses of slow convergence speed, easily falling into local minimum and frequent occurrence of oscillation in learning process of traditional algorithms, eliminating bias caused by arbitrary individual experts, improving efficiency of bid evaluation, and providing effective reference and basis for bid evaluation activities of general engineering projects.
\end{abstract}

Keywords: Artificial neural network, Bid evaluation, BP neural network, Momentum BP algorithm, Order relation analysis.

\section{INTRODUCTION}

As the continuous development of engineering projects bidding system, bid evaluation methods have become a main focus of public attention. Bid evaluation is the core of the bidding and the premise of scientific decision-making, thus playing a very important role in bidding. It is essential to follow the principles of openness, fairness, impartiality and good faith, and carry out a synthetic assessment of all aspects of the bidders, such as project quotation, project duration, project quality, material consumption, construction technology, management level, corporate reputation and preferential terms, on the premise of satisfying the substantive requirements of bid documents as well as possible.

Since the actual operation of bid evaluation faces many issues, especially bid evaluation methods lacking research in theory and lacking experience in practice. Therefore, it is of great theoretic and realistic significance to study bid evaluation methods for engineering projects and establish a scientific, practical and feasible assessment model. Currently, the most universal decision-making method of bid evaluation is comprehensive scoring method or reasonable low price method [1, 2], and analytic hierarchy process $[3,4]$ or fuzzy comprehensive assessment [5] is also used for bid evaluation $[6,7]$, but all these methods are difficult to avoid bias caused by arbitrary individual experts.

*Address correspondence to this author at the School of Management, Hubei University of Technology, Wuhan, Hubei, 430068, P.R. China; Tel: 15527903758; E-mail: hgzhangyi@163.com
In order to solve the problem mentioned above, this paper tried to apply the theory and method of artificial neural network (ANN) [8-10] and established a decision-making model of bid evaluation for engineering projects based on back propagation neural network (BPNN). BP neural network was trained and tested by the learning sample that was attained by using order relation analysis method $\left(\mathrm{G}_{1}-\right.$ method). At the same time, we used the momentum BP algorithm to solve the model. The case was given to conduct a comprehensive and objective assessment of bidders and provide a scientific and practical approach for decision-makers. The results show that the model is feasible and reasonable, possessing high abilities of self-organization, self-adaptation and self-learning and strong function of fault-tolerance, avoiding weaknesses of slow convergence speed, easily falling into local minimum and frequent occurrence of oscillation in learning process of traditional algorithms, eliminating bias caused by arbitrary individual experts, improving efficiency of bid evaluation and providing a new approach and idea for decision-makers of engineering projects.

The general outline of the paper is organized as follows: the first part is explaining and summarizing the background and purpose of the research; the second part is establishing a decision-making model of bid evaluation based on BP neural network and adopting the momentum BP algorithm to solve the model; the third part is obtaining the learning sample that was attained by using order relation analysis method $\left(\mathrm{G}_{1^{-}}\right.$ method); the fourth part is using a case study to confirm the feasibility and reliability of the decision-making model of bid evaluation; the fifth part is the conclusion and discussion. 


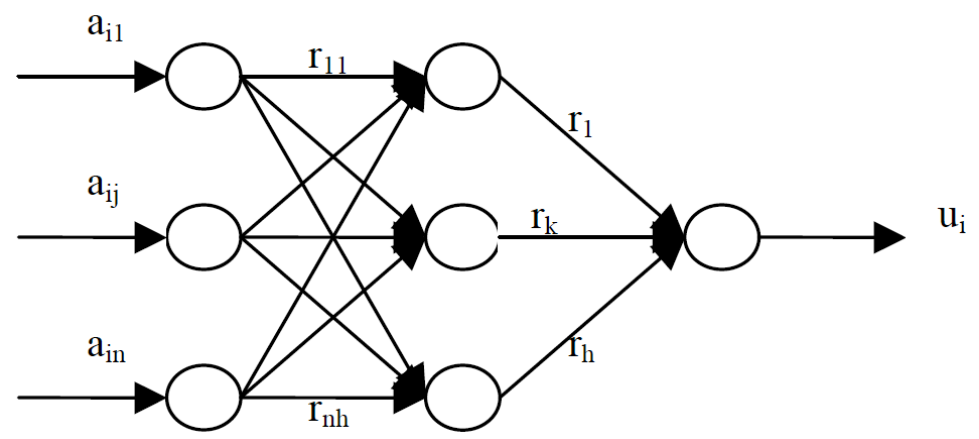

Fig. (1). Topological structure of BP neural network.

\section{ESTABLISHING A DECISION-MAKING MODEL OF BID EVALUATION BASED ON BACK PROPA- GATION NEURAL NETWORK}

\subsection{Constructing Bid Evaluation Model Based on BP Neural Network}

Back propagation neural network $[11,12]$ is a multi-layer network model of feedback type proposed by the famous researcher Rumelhart [13] in 1985, which is one of the most widely used artificial neural network models. It consists of input layer, hidden layer and output layer. All nodes between two adjacent layers implement weight link and there is no link between nodes of the same layer.

This paper adopted a three-layer BP neural network model of multi-inputs and single-output. The essential idea is taking the assessment index values of the sample as the inputs of BP neural network model, taking the assessment result as the output of BP neural network model, training this network with enough samples, acquiring knowledge structure, cognitive ability, experience level and personal preference of bid evaluation experts by continuously adjusting link weights and thresholds. So link weights and thresholds are internal expression of correct knowledge obtained by BP network model through self-learning. We can obtain the assessment result of each bidder according to the assessment index values of each bidder through the trained BP network model, and then we can sort various bidders. The figure of topological structure is shown in Fig. (1).

In Fig. (1), the number of input layer nodes is $n$, which is also the number of assessment indexes. The number of output layer nodes is one, which is also the number of assessment result. The number of hidden layer nodes is $h$, which can be calculated by adopting the empirical formula " $h=\sqrt{n+s}+q$ ", in which, $s$ is the number of output layer nodes, obviously $s=1$, and $q$ is the constant among one to ten.

Assume $A_{i}=\left(a_{i 1}, \cdots, a_{i j}, \cdots, a_{i n}\right)(i=1,2, \cdots m)$ as assessment index values vector of the $i$ th sample on the assessment indexes set $B=\left\{B_{1}, \cdots, B_{j}, \cdots, B_{n}\right\}$ and $m$ is the number of samples, so we can construct the matrix $A=\left(A_{1}, \cdots\right.$, $\left.A_{i}, \cdots, A_{m}\right)^{T}=\left(a_{i j}\right)_{m \times n}$ by $m$ samples. Assume $r_{j k}(j=1$, $2, \cdots n ; k=1,2, \cdots h)$ is the link weight from the $j$ th node of input layer to the $k$ th node of hidden layer. Assume $u_{i k}$ is the output of the $k$ th node of hidden layer of the $i$ th sample. Assume $r_{k}$ is the link weight from the $k$ th node of hidden layer to the output layer. Assume $u_{i}$ is the output of the $i$ th sample.

Each node is corresponding to a function $F(x)$ and a threshold. For input layer nodes, the output of which is the same as the input, namely $F(x)=x$ and the threshold value is zero; for hidden and output layer nodes, we usually choose the Sigmoid function, namely $F(x)=1 /\left(1+e^{-x}\right)$. Assume $g_{k}$ is the threshold of the $k$ th node of hidden layer and $g$ is the threshold of the output layer node, so $u_{i k}=F\left(\sum_{j=1}^{n} r_{j k} a_{i j}-g_{k}\right)$ and $u_{i}=F\left(\sum_{k=1}^{h} r_{k} u_{i k}-g\right)$.

\subsection{Momentum BP Algorithm}

BP algorithm is a kind of supervised learning algorithm. The basic idea is inputting the learning samples and the input information reaches the output layer from the input layer via the hidden layer. If the error doesn't reach the acceptable level, it will turn to back propagation, making the actual output as close as possible to the expected output by adjusting link weights and thresholds and continuously iterating. If the error reaches the acceptable level, the training is completed, and then we can save link weights and thresholds of the network.

Assume $v_{i}$ as the expected output. Assume $t$ as the iteration time. Assume $u_{i}(t)$ as the actual output of the $t$ th iteration time of the $i$ th sample. Assume $D_{i}(t)$ as the error function of the $t$ th iteration time of the $i$ th sample. Assume $D(t)$ 
as the total error function of the $t$ th iteration time, this paper definite

$$
D(t)=\sum_{i=1}^{m} D_{i}(t)=\sum_{i=1}^{m}\left[u_{i}(t)-v_{i}\right]^{2} / 2 \text {. }
$$

Traditional BP algorithm has several disadvantages, such as slow convergence speed, easily falling into local minimum and frequent occurrence of oscillation in learning process. In order to overcome shortages mentioned above, there appeared a lot of improved algorithms, such as the momentum BP algorithm, the elastic algorithm, the variable gradient algorithm, the quasi-Newton algorithm, the LM algorithm and so on. This paper adopted the momentum BP algorithm [14-15]. In neural network tool-box of MATLAB7.0, the training function is traingdm, which introduces the momentum factor $\lambda(0<\lambda<1)$ [16] on the basis of the gradient descent algorithm, using the inertial effect to curb possible oscillation and playing a smooth effect. At the same time, the role of the momentum factor lies in memory of the change of previous link weight values or threshold values, so that we can use the higher learning rate $\theta$ to improve the speed of learning [17].

The modified formula of link weight values and threshold values of momentum BP algorithm is as follows:

$y(t+1)=y(t)+\theta z(t)+\lambda[y(t)-y(t-1)]$

In which, $y(t)$ expresses link weights or thresholds of the $t$ th iteration time, $\theta(0<\theta<1)$ is the learning rate, $z(t)=\frac{\partial D(t)}{\partial y(t)}$ expresses the partial derivative of the error to each link weight or threshold of the $t$ th iteration time.

For output layer (The number of output layer nodes is one),

$r_{k}(t+1)=r_{k}(t)+\theta \sum_{i=1}^{m} \varepsilon_{i} u_{i k}+\lambda\left[r_{k}(t)-r_{k}(t-1)\right]$
$g(t+1)=g(t)+\theta \sum_{i=1}^{m} \varepsilon_{i}+\lambda[g(t)-g(t-1)]$.

For hidden layer,

$r_{j k}(t+1)=r_{j k}(t)+\theta \sum_{i=1}^{m} \varepsilon_{i k} a_{i j}+\lambda\left[r_{j k}(t)-r_{j k}(t-1)\right]$

$g_{k}(t+1)=g_{k}(t)+\theta \sum_{i=1}^{m} \varepsilon_{i k}+\lambda\left[g_{k}(t)-g_{k}(t-1)\right]$.

In which, $\varepsilon_{i}$ is the delta error of back propagation of the output layer and $\varepsilon_{i}=\left(v_{i}-u_{i}\right) u_{i}\left(1-u_{i}\right) ; \varepsilon_{i k}$ is the delta error of back propagation of the hidden layer and $\varepsilon_{i k}=\varepsilon_{i} r_{k} u_{i k}\left(1-u_{i k}\right)$.
3. OBTAINING THE SAMPLE BY USING ORDER RELATION ANALYSIS METHOD (G (GETHOD) $_{1}$-METHO

\subsection{Determining Index Weights by Using Order Relation Analysis Method $\left(G_{1}-\right.$ Method)}

In general the determination of index weights adopts the AHP, $\mathrm{G}_{1}$-method, $\mathrm{G}_{2}$-method and Set-valued iterative method. This paper uses the $G_{1}$-method [18]. This method is not only simple and intuitive, but also it can be used without constructing judgment matrix and testing consistency. The concrete steps are as follows.

\section{1) Determining the order relation}

First of all, experts select the index that he or she considered as the most important unique index from the assessment indexes set $B=\left\{B_{1}, B_{2}, \cdots, B_{j}, \cdots, B_{n}\right\}(j=1,2, \cdots n)$ and recorded as $B_{1}^{*}$, then choose the index that he or she considered as the most important unique index from remaining $n-1$ indexes and recorded as $B_{2}^{*}$, and so on, after the selection of $n-1$ times, the remaining assessment index is recorded as $B_{n}^{*}$, so that we can determine the only order relation: $B_{1}^{*} \succ B_{2}^{*} \succ \cdots \succ B_{p-2}^{*} \succ B_{p-1}^{*} \succ B_{p}^{*} \succ \cdots \succ B_{n}^{*}$.

2) Obtaining the comparison judgment of relative importance between $B_{p-1}^{*}$ and $B_{p}^{*}$

It is assumed that experts make rational judgment on the ratio $w_{p-1}^{*} / w_{p}^{*}$ of importance degree of the assessment index $B_{p-1}^{*}$ and $B_{p}^{*}$ :

$d_{p}=\frac{w_{p-1}^{*}}{w_{p}^{*}}(p=n, n-1, \cdots, 3,2)$

The value of $d_{p}$ can refer to the following Table 1.

3) Calculating the weight coefficients

It is obvious that $w_{p-2}^{*}>w_{p}^{*}$, and because $w_{p-1}^{*}>0$, so $\frac{w_{p-2}^{*}}{w_{p-1}^{*}}>\frac{w_{p}^{*}}{w_{p-1}^{*}}$, namely $d_{p-1}>\frac{1}{d_{p}}$.

On account of

$$
\prod_{j=p}^{n} d_{j}=\frac{w_{p-1}^{*}}{w_{p}^{*}} \times \frac{w_{p}^{*}}{w_{p+1}^{*}} \times \frac{w_{p+1}^{*}}{w_{p+2}^{*}} \times \cdots \times \frac{w_{n-2}^{*}}{w_{n-1}^{*}} \times \frac{w_{n-1}^{*}}{w_{n}^{*}}=\frac{w_{p-1}^{*}}{w_{n}^{*}}
$$

The sum of the formula mentioned above from $p=2$ to $p=n$ is as follows:

$\sum_{p=2}^{n}\left(\prod_{j=p}^{n} d_{j}\right)=\sum_{p=2}^{n} \frac{w_{p-1}^{*}}{w_{n}^{*}}=\frac{1}{w_{n}^{*}}\left(w_{1}^{*}+w_{2}^{*}+\cdots w_{n-1}^{*}\right)$ 
Table 1. Assignment reference of $d_{p}$.

\begin{tabular}{|c|c|}
\hline$d_{p}$ & Meaning \\
\hline \hline $\mathbf{1 . 0}$ & The former is as important as the latter \\
\hline $\mathbf{1 . 2}$ & The former is slightly more important than the latter \\
\hline $\mathbf{1 . 4}$ & The former is obvious more important than the latter \\
\hline $\mathbf{1 . 6}$ & The former is very more important than the latter \\
\hline $\mathbf{1 . 8}$ & The former is extreme more important than the latter \\
\hline
\end{tabular}

$=\frac{1}{w_{n}^{*}}\left(\sum_{p=1}^{n} w_{p}^{*}-w_{n}^{*}\right)$

and because $\sum_{p=1}^{n} w_{p}^{*}=1$

So $\sum_{p=2}^{n}\left(\prod_{j=p}^{n} d_{j}\right)=\frac{1}{w_{n}^{*}}\left(1-w_{n}^{*}\right)=\frac{1}{w_{n}^{*}}-1$

Therefore $w_{n}^{*}=\left[1+\sum_{p=2}^{n}\left(\prod_{j=p}^{n} d_{j}\right)\right]^{-1}$

According to equation (1), we can obtain:

$w_{p-1}^{*}=d_{p} w_{p}^{*}(p=n, n-1, \cdots, 3,2)$

So according to equation (2) and equation (3), we can obtain the index weights vector $W^{*}=\left(w_{1}^{*}, w_{2}^{*}, \cdots, w_{n}^{*}\right)^{T}$ of the assessment indexes set $B^{*}=\left\{B_{1}^{*}, B_{2}^{*}, \cdots, B_{n}^{*}\right\}$, so that we can obtain the index weights vector $W=\left(w_{1}, w_{2}, \cdots, w_{n}\right)^{T}$ of the assessment indexes set $B=\left\{B_{1}, B_{2}, \cdots, B_{j}, \cdots, B_{n}\right\} \quad(j=1$, $2, \cdots n)$.

\subsection{Calculating the Total Score of Each Bidder}

Assume $B=\left\{B_{1}, B_{2}, \cdots, B_{j}, \cdots, B_{n}\right\} \quad(j=1,2, \cdots n)$ as assessment indexes set and $n$ is the number of assessment indexes. Assume $C=\left\{C_{1}, C_{2}, \cdots, C_{f}\right\}$ as assessment grades set and $f$ is the number of assessment grades. It describes the state of assessment indexes and is usually divided into 3 to 5 grades [19]. Bid evaluation experts should score all assessment indexes of each bidder independently and impartially on the basis of comparison of various bidders. This paper ordered $\mathrm{C}=$ \{eligible, moderate, good, excellent $\}$ and it's corresponding scores interval set is $\left.\left[e_{2}, e_{3}\right),\left[e_{3}, e_{4}\right)\right\}\left[e_{2}, e_{3}\right)$, $\left.\left[e_{3}, e_{4}\right)\right\}=\{[0.6,0.7),[0.7,0.8),[0.8,09),[0.9,1.0)\} . \mathrm{We}$ can obtain the total score of each bidder by multiplying the row vector of index scores and the column vector of index weights.

\section{CASE STUDY}

\subsection{Constructing Assessment Indexes System}

The selection of assessment indexes is a very important work. In actual process of comprehensive assessment, it is common to use methods of Delphi, minimum mean square error, mini-max dispersion and correlative coefficient to select indicators [20]. This paper used the method of Delphi to get the assessment indexes system, which consists of six assessment indexes of project quotation, project duration, project quality, construction technology, management organization and corporate reputation, namely $\mathrm{B}=$ \{project quotation $\left(\mathrm{B}_{1}\right)$, project duration $\left(\mathrm{B}_{2}\right)$, project quality $\left(\mathrm{B}_{3}\right)$, construction technology $\left(\mathrm{B}_{4}\right)$, management organization $\left(\mathrm{B}_{5}\right)$, corporate reputation $\left(\mathrm{B}_{6}\right)$ \}.

\subsection{Determining Index Weights by Using Order Relation Analysis Method ( $G_{1}$-Method)}

It is assumed that experts believe that there being the order relation among assessment indexes as follows:

$B_{1} \succ B_{3} \succ B_{2} \succ B_{4} \succ B_{5} \succ B_{6}$

Namely $B_{1}^{*} \succ B_{2}^{*} \succ B_{3}^{*} \succ B_{4}^{*} \succ B_{5}^{*} \succ B_{6}^{*}$

Meanwhile, experts make rational judgment on the ratio of importance degree between every two adjacent assessment indexes as follows:

$d_{2}=\frac{w_{1}^{*}}{w_{2}^{*}}=1.6, d_{3}=\frac{w_{2}^{*}}{w_{3}^{*}}=1.4, d_{4}=\frac{w_{3}^{*}}{w_{4}^{*}}=1.7, d_{5}=\frac{w_{4}^{*}}{w_{5}^{*}}=1.5$,

$d_{6}=\frac{w_{5}^{*}}{w_{6}^{*}}=1.2$

so that we can obtain

$\prod_{j=2}^{6} d_{j}=d_{2} d_{3} d_{4} d_{5} d_{6}=6.8544, \prod_{j=3}^{6} d_{j}=d_{3} d_{4} d_{5} d_{6}=4.284$, 


$$
\begin{aligned}
& \prod_{j=4}^{6} d_{j}=d_{4} d_{5} d_{6}=3.06 \\
& \prod_{j=5}^{6} d_{j}=d_{5} d_{6}=1.8, \prod_{j=6}^{6} d_{j}=d_{6}=1.2 .
\end{aligned}
$$

and then we can obtain

$$
\begin{aligned}
& \sum_{p=2}^{6}\left(\prod_{j=p}^{6} d_{j}\right)=\prod_{j=2}^{6} d_{j}+\prod_{j=3}^{6} d_{j}+\prod_{j=4}^{6} d_{j}+\prod_{j=5}^{6} d_{j}+\prod_{j=6}^{6} d_{j} \\
& =d_{2} d_{3} d_{4} d_{5} d_{6}+d_{3} d_{4} d_{5} d_{6}+d_{4} d_{5} d_{6}+d_{5} d_{6}+d_{6}=17.1984
\end{aligned}
$$

According to equation (2), we can obtain:

$$
w_{6}^{*}=\left[1+\sum_{p=2}^{6}\left(\prod_{j=p}^{6} d_{j}\right)\right]^{-1}=(1+17.1984)^{-1}=0.055
$$

According to equation (3), we can obtain:

$$
\begin{aligned}
& w_{5}^{*}=d_{6} w_{6}^{*}=0.066, w_{4}^{*}=d_{5} w_{5}^{*}=0.099, w_{3}^{*}=d_{4} w_{4}^{*}=0.168, \\
& w_{2}^{*}=d_{3} w_{3}^{*}=0.235, w_{1}^{*}=d_{2} w_{2}^{*}=0.377 .
\end{aligned}
$$

So the weight coefficients are as follows: $w_{1}=w_{1}^{*}=0.377 \quad, \quad w_{2}=w_{3}^{*}=0.168, \quad w_{3}=w_{2}^{*}=0.235$, $w_{4}=w_{4}^{*}=0.099, w_{5}=w_{5}^{*}=0.066, w_{6}=w_{6}^{*}=0.055$.

So that we can obtain the index weights vector as follows:

$$
\begin{aligned}
& W=\left(w_{1}, w_{2}, \cdots, w_{n}\right)^{T}=\left(w_{1}, w_{2}, w_{3}, w_{4}, w_{5}, w_{6}\right)^{T} \\
& =(0.377,0.168,0.235,0.099,0.066,0.055)^{T}
\end{aligned}
$$

\subsection{Calculating the Total Score of Each Bidder}

As mentioned previously, this paper ordered $\mathrm{C}=\{$ eligible, moderate, good, excellent $\}$ and it's corresponding scores interval set is $E=\left\{\left[e_{0}, e_{1}\right),\left[e_{1}, e_{2}\right),\left[e_{2}, e_{3}\right),\left[e_{3}, e_{4}\right)\right\}=\{[0.6,0.7)$, $[0.7,0.8),[0.8,09),[0.9,1.0)\}$. The index weights vector that we have obtained in the previous section is $W=(0.377,0.168,0.235,0.099,0.066,0.055)^{T}$. We can obtain the total score of each bidder by multiplying the row vector of index scores and the column vector of index weights. This paper chose twenty-four bidders. The score of every assessment index and the total score of each bidder are shown in Table $\mathbf{2}$ as follows.

\subsection{Training and Testing BP Network}

We take scores of six assessment indexes of project quotation $\left(\mathrm{B}_{1}\right)$, project duration $\left(\mathrm{B}_{2}\right)$, project quality $\left(\mathrm{B}_{3}\right)$, construction technology $\left(\mathrm{B}_{4}\right)$, management organization $\left(\mathrm{B}_{5}\right)$, corporate reputation $\left(\mathrm{B}_{6}\right)$ as the inputs of back propagation neural network, so the number of input layer nodes is six, namely $n=6$. We take the total score as the output of back propagation neural network, so the number of output layer nodes is one. We determine the number of hidden layer nodes is ten according to experience and repeated test, namely $h=10$. We divide the data in Table 2 into two parts: the first 18 sets of data are taken as the learning samples which are used to train the neural network and remaining 6 sets of data are taken as the checking samples which are used to test the neural network. Assume the learning precision $\delta=10^{-6}$. When the neural network is trained to a certain number of times, we will get a satisfactory precision. The learning results are shown in Table $\mathbf{3}$ as follows.

From the Table 3, we find errors between the training results and the expected output is very small and the maximum relative error is $0.188 \%$, so the learning results of back propagation neural network are ideal. After completing the training and entering the last 6 groups of calibration data into the trained back propagation neural network, we will get the comprehensive assessment result of the back propagation neural network as shown in Table 4.

From the Table 4, we find the maximum relative error is $0.92 \%$ between the training results and the expected outputs obtained by the neural networks and the simulation ordering is in accordance with the experts ordering. Thus it can be seen that using the BP neural network to access knowledge and experience of bid evaluation experts and using the trained BP neural network to assess the similar engineering projects is completely feasible.

\section{CONCLUSION}

1) Decision-Making of bid evaluation model for engineering project based on BP neural network obtained knowledge and experience of bid evaluation experts by learning several existing samples. When facing the similar engineering projects which need to be evaluated, we can get assessment results as long as we enter the corresponding assessment index values into the trained BP neural network and launch the network. The learning samples of the BP neural network are obtained from assessment results of order relation analysis method $\left(\mathrm{G}_{1}\right.$-method), so the decisionmaking model of bid evaluation for engineering projects based on BP neural network can not completely replace order relation analysis method $\left(\mathrm{G}_{1}\right.$-method). In particular, compared with the traditional AHP method, this method is more convenient and intuitive without constructing judgment matrix and checking consistency.

2) BP neural network possess high abilities of selforganization, self-adaptation and self-learning and strong function of fault-tolerance, avoiding weaknesses of slow convergence speed, easily falling into local minimum and frequent occurrence of oscillation in learning process of traditional algorithms, eliminating bias of arbitrary individual experts, ensuring the accuracy of the evaluation results and improving efficiency of bid evaluation.

3) Determining the number of the hidden layer nodes is very important. Too many nodes can cause too long learning time, too little nodes can lead to poor fault-tolerance and poor ability of identifying the samples without learning, so we must design the numbers of the hidden layer nodes based on comprehensive comparison of various factors. Meanwhile, the condition of trapping in local minimum may 
Table 2. Score of every index and total score of each bidder.

\begin{tabular}{|c|c|c|c|c|c|c|}
\hline Bidder & 1 & 2 & 3 & 4 & 5 & 6 \\
\hline $\mathrm{B}_{1}$ & 0.90 & 0.70 & 0.80 & 0.75 & 0.90 & 0.90 \\
\hline $\mathrm{B}_{2}$ & 0.70 & 0.60 & 0.90 & 0.70 & 0.90 & 0.95 \\
\hline $\mathrm{B}_{3}$ & 0.70 & 0.80 & 0.80 & 0.70 & 0.80 & 0.85 \\
\hline $\mathrm{B}_{4}$ & 0.65 & 0.70 & 0.85 & 0.90 & 0.80 & 0.85 \\
\hline $\mathrm{B}_{5}$ & 0.90 & 0.80 & 0.70 & 0.80 & 0.85 & 0.90 \\
\hline $\mathrm{B}_{6}$ & 0.75 & 0.70 & 0.75 & 0.65 & 0.80 & 0.80 \\
\hline TS & 0.7864 & 0.7133 & 0.8124 & 0.7425 & 0.8578 & 0.8862 \\
\hline Bidder & 7 & 8 & 9 & 10 & 11 & 12 \\
\hline $\mathrm{B}_{1}$ & 0.70 & 0.60 & 0.80 & 0.75 & 0.65 & 0.90 \\
\hline $\mathrm{B}_{2}$ & 0.85 & 0.70 & 0.70 & 0.85 & 0.80 & 0.90 \\
\hline $\mathrm{B}_{3}$ & 0.90 & 0.70 & 0.80 & 0.80 & 0.80 & 0.80 \\
\hline $\mathrm{B}_{4}$ & 0.80 & 0.80 & 0.70 & 0.65 & 0.90 & 0.70 \\
\hline $\mathrm{B}_{5}$ & 0.80 & 0.80 & 0.60 & 0.90 & 0.85 & 0.75 \\
\hline $\mathrm{B}_{6}$ & 0.90 & 0.80 & 0.80 & 0.70 & 0.75 & 0.80 \\
\hline TS & 0.7997 & 0.6843 & 0.7601 & 0.7758 & 0.7539 & 0.8413 \\
\hline Bidder & 13 & 14 & 15 & 16 & 17 & 18 \\
\hline $\mathrm{B}_{1}$ & 0.60 & 0.60 & 0.70 & 0.70 & 0.80 & 0.85 \\
\hline $\mathrm{B}_{2}$ & 0.70 & 0.60 & 0.60 & 0.70 & 0.90 & 0.85 \\
\hline $\mathrm{B}_{3}$ & 0.80 & 0.70 & 0.80 & 0.65 & 0.85 & 0.80 \\
\hline $\mathrm{B}_{4}$ & 0.90 & 0.70 & 0.85 & 0.70 & 0.85 & 0.85 \\
\hline $\mathrm{B}_{5}$ & 0.85 & 0.65 & 0.90 & 0.60 & 0.85 & 0.80 \\
\hline $\mathrm{B}_{6}$ & 0.90 & 0.60 & 0.75 & 0.65 & 0.90 & 0.80 \\
\hline TS & 0.7265 & 0.6367 & 0.7375 & 0.6789 & 0.8423 & 0.8322 \\
\hline Bidder & 19 & 20 & 21 & 22 & 23 & 24 \\
\hline $\mathrm{B}_{1}$ & 0.80 & 0.90 & 0.60 & 0.65 & 0.65 & 0.90 \\
\hline $\mathrm{B}_{2}$ & 0.85 & 0.90 & 0.80 & 0.70 & 0.60 & 0.70 \\
\hline $\mathrm{B}_{3}$ & 0.85 & 0.85 & 0.90 & 0.70 & 0.70 & 0.70 \\
\hline $\mathrm{B}_{4}$ & 0.80 & 0.85 & 0.70 & 0.80 & 0.70 & 0.60 \\
\hline $\mathrm{B}_{5}$ & 0.80 & 0.80 & 0.90 & 0.85 & 0.70 & 0.70 \\
\hline $\mathrm{B}_{6}$ & 0.85 & 0.80 & 0.70 & 0.85 & 0.65 & 0.70 \\
\hline TS & 0.8229 & 0.8712 & 0.7393 & 0.7092 & 0.6616 & 0.7655 \\
\hline
\end{tabular}


Table 3. Learning result.

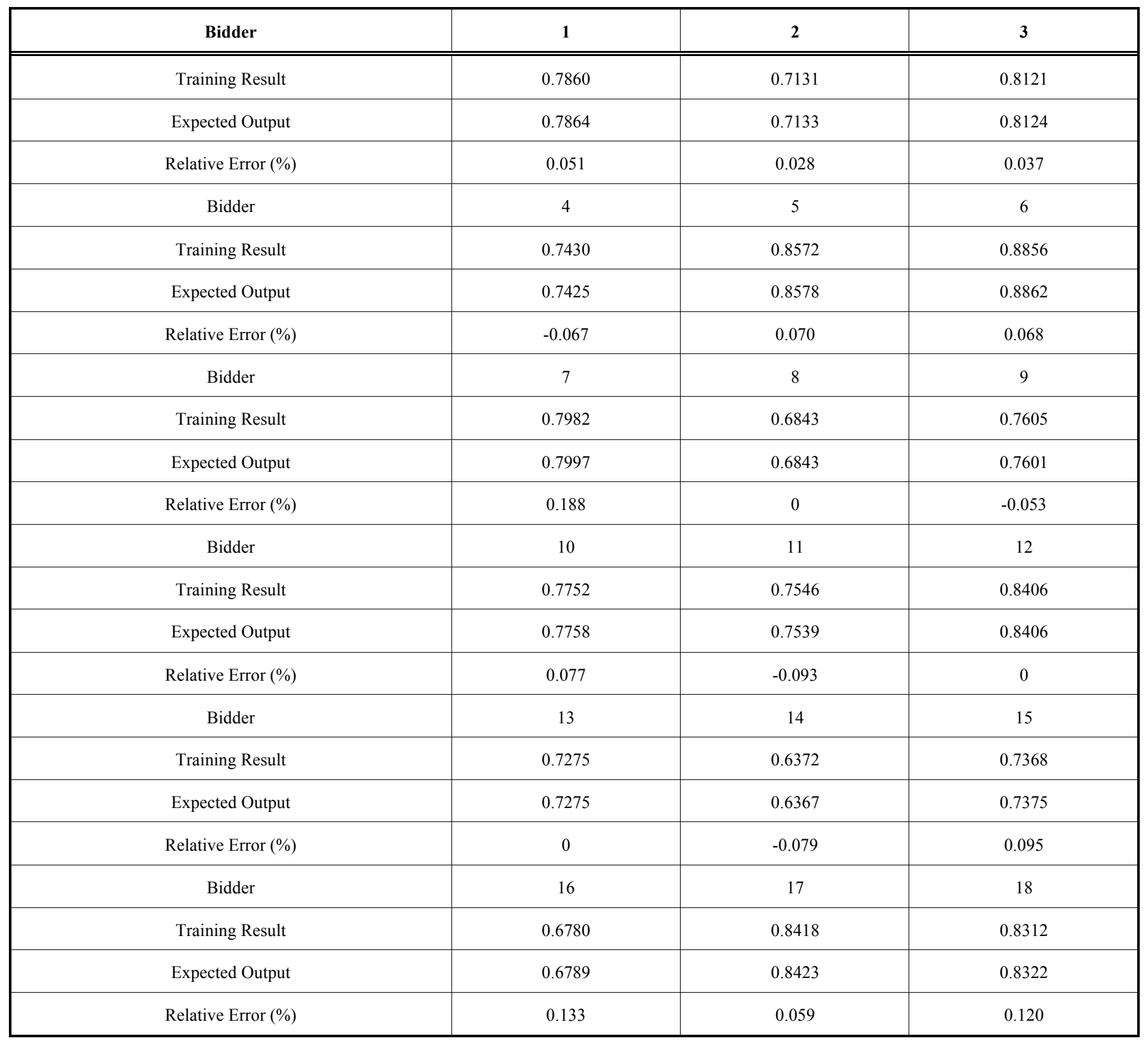

Table 4. Result checking.

\begin{tabular}{|c|c|c|c|}
\hline Bidder & $\mathbf{1 9}$ & $\mathbf{2 0}$ \\
\hline \hline Testing Result & 0.8202 & 0.8658 & 0.7325 \\
\hline Expected Output & 0.8229 & 0.8712 & 0.7393 \\
\hline Relative Error (\%) & 0.328 & 1 & 4 \\
\hline Simulation Sort & 2 & 1 & 4 \\
\hline Expert Sort & 2 & 320 \\
\hline
\end{tabular}


Table 4. contd...

\begin{tabular}{|c|c|c|c|}
\hline Bidder & $\mathbf{2 2}$ & $\mathbf{2 3}$ & $\mathbf{2 4}$ \\
\hline Testing Result & 0.7092 & 0.6562 & 0.7687 \\
\hline Expected Output & 0.7092 & 0.6616 & 0.7655 \\
\hline Relative Error (\%) & 0 & 0.816 & -0.418 \\
\hline Simulation Sort & 5 & 6 & 3 \\
\hline Expert Sort & 5 & 6 & 3 \\
\hline
\end{tabular}

occur in the training process of BP neural network, so we can get global minimum by changing its initial value and training for many times.

\section{CONFLICT OF INTEREST}

The author confirms that this article content has no conflict of interest.

\section{ACKNOWLEDGEMENTS}

This work is supported by the Youth Fund Program of Humanities and Social Science Research of Ministry of Education of China (Program name: Research on Index System and Decision-Making Method of Bid Evaluation for Engineering Projects Based on D-S Evidence Theory from the Perspective of Green; Grant No.14YJC630201).

\section{REFERENCES}

[1] H. L. Zou, S. S. Zhao, and L. X. Zhang, "Evaluation method for engineering tenders based on synthetic analysis of quantitative and qualitative indexes", Journal of the China Railway Society, vol. 22, no. 6, pp. 112-115, 2000.

[2] C. S. Chen, and L. Shen, "Bid Evaluation Expert Guidelines," Electronics Industry Press: Beijing 2006.

[3] T. L. Satty, "The Analytic Hierarchy Process," McGraw-Hill: New York, 1980.

[4] T. L. Satty, and L. G. Vargas, "Uncertainty and rank order in the analytic hierarchy process", European Journal of Operational Research, vol. 32, no. 1, pp. 107-117, 1987.

[5] J. C. Wang, "Multi-indexes fuzzy comprehensive judgment", Journal of Kunming University of Technology, vol. 23, no. 4, pp. 69-71, 1998.
[6] L. T. Shen, and Q. M. Li, "Application of analytic hierarchy process in construction engineering bid evaluation", Construction Technology, vol. 34, pp. 64-66, 2005.

[7] B. Pan, and D. R. Zhang, "Evaluation for government purchasing bidding projects: A comprehensive analysis on the basis of vague mathematics", Systems Engineering, vol. 25, pp. 97-100, 2007.

[8] L. Q. Han, “Artificial Neural Network Tutorial," Beijing University of Posts and Telecommunications Press: Beijing, 2006.

[9] R. Ma, "Artificial Neural Network Theory," Machinery Industry Press: Beijing, 2010.

[10] S. Q. Ding, and L. H. Guo, "Artificial Neural Network Foundation," Harbin Engineering University Press: Harbin 2008.

[11] C. H. He, Q. Y. Huang, S. F. Shen, and F. Wang, "Forest fuel loading estimates based on a back propagation neutral network", Journal of Tsinghua University, Science and Technology, vol. 51, no. 2, pp. 230-233, 2011.

[12] Q. N. Han, Y. L. Hao, Z. P. Liu, and R. Wang, "Prediction of the angular velocity of GFSINS by BP neural network", Journal of Huazhong University of Science and Technology (Nature Science Edition), vol. 39, no. 3, pp. 115-119, 2011.

[13] A. S. Weigend, B. A. Huberman, and D. E. Rumelhart, "Predicting the future: a connectionist approach", International Journal of Neural Systems, vol. 1, no. 3, pp. 193-209, 1990.

[14] D. F. Zhang, Simulation and Application of MATLAB Neural Network, Electronics Industry Press: Beijing, 2009.

[15] M. X. He, and L. J. Wang, "Momentum back propagation for the settlement prediction of sub-grade construction", Geotechnical Engineering Technique, vol. 20, no. 1, pp. 17-20, 2006.

[16] Y. M. Ma, and Y. H. Wu, "Influence of momentum gene on BP algorithms", Journal of the Central University for Nationalities (Natural Sciences Edition), vol. 17, no. 4, pp. 35-40, 2008.

[17] X. S. Tan, and T. J. Zhou, "Methods to improve BP neural network", Journal of Huaihua University, vol. 25, no. 2, pp. 126-130, 2006.

[18] Y. J. Guo, The Theory, Method and Application of Comprehensive Evaluation, Science Press: Beijing, 2007.

[19] D. Du, Q. H. Pang, and Y. Wu, Modern Comprehensive Evaluation Method and Case Selection, Tsinghua University Press: Beijing, 2008.

[20] Z. P. Xu, and W. Wu, Multiple Attribute Decision Making Theory and Methods, Tsinghua University Press: Beijing, 2006. 